\title{
THE IMPERFECTIVE PARADOX AND TRAJECTORY-OF-MOTION EVENTS *
}

\author{
Michael White \\ Department of Computer and Information Science \\ University of Pennsylvania \\ Philadelphia, PA, USA \\ mwhite@linc.cis . upenn .edu
}

\begin{abstract}
In the first part of the paper, I present a new treatment of THE IMPERFECTIVE PARADOX (Dowty 1979) for the restricted case of trajectoryof-motion events. This treatment extends and refines those of Moens and Steedman (1988) and Jackendoff (1991). In the second part, I describe an implemented algorithm based on this treatment which determines whether a specified sequence of such events is or is not possible under certain situationally supplied constraints and restrictive assumptions.
\end{abstract}

\section{Introduction}

Bach (1986:12) summarizes THE IMPERFECTIVE PARADOX (Dowty 1979) as follows: "...how can we characterize the meaning of a progressive sentence like (1a) [17] on the basis of the meaning of a simple sentence like (1b) [18] when (1a) can be true of a history without (1b) ever being true?"

(1a) John was crossing the street.

(1b) John crossed the street.

Citing parallels in the nominal domain, Bach goes on to point out that this puzzle is seemingly much more general, insofar as it appears whenever any sort of partitive is employed. In support of this view, we may observe that the start v-ing construction exhibits the same behavior:

(2a) John started jogging to the museum.

(2b) John jogged to the museum.

Here we see that (2a) does not entail (2b) - while (2b) asserts the occurrence of an entire event of John jogging to the museum, (2a) only asserts the

*The author gratefully acknowledges the helpful comments of Mark Steedman, Jeff Siskind, Christy Doran, Matthew Stone, and the anonymous referees, as well as the support of DARPA N00014-90-J1863, ARO DAAL03-89-C-0031, NSF IRI 90-16592, Ben Franklin 91S.3078C-1. occurrence of the beginning of such an event, leaving open the existential status of its completion.

Capitalizing on Bach's insight, I present in the first part of the paper a new treatment of the imperfective paradox which relies on the possibility of having actual events standing in the part-of relation to hypothetical super-events. This treatment extends and refines those of Moens and Steedman (1988) and Jackendoff (1991), at least for the restricted case of trajectory-of-motion events. ${ }^{1}$ In particular, the present treatment correctly accounts not only for what (2a) fails to entail - namely, that John eventually reaches the museum - but also for what (2a) does in fact entail - namely, that John follows (by jogging) at least an initial part of a path that leads to the museum. In the second part of the paper, I briefly describe an implemented algorithm based on this theoretical treatment which determines whether a specified sequence of trajectory-of-motion is or is not possible under certain situationally supplied constraints and restrictive assumptions.

\section{Theory}

The present treatment builds upon the approach to aspectual composition developed in White (1993), a brief sketch of which follows. White (1993) argues that substances, processes and other such entities should be modeled as abstract kinds whose realizations (things, events, etc.) vary in amount. ${ }^{2}$ This is accomplished formally through the use of an order-sorted logic with an axiomatized collection of binary relations. The intended sort hierarchy is much like those of Eberle (1990) and Jackendoff (1991); in particular, both substances and things are taken to be subsorts of the material entities, and similarly

\footnotetext{
'These are elsewhere called 'directed-motion' events.

${ }^{2}$ This move is intended to resolve certain empirical and computational problems with the view of referential homogeneity espoused by Krifka (1992) and his predecessors.
} 
both processes and events are taken to be subsorts of the non-stative eventualities. What is new is the axiomatization of Jackendoff's composed-of relation (comp) - which effects the aforementioned kind-to-realization mapping - in terms of Krifka's (1992) part-of relation (ㄷ). Of particular interest is the following subpart closure property:

(3) $\forall x y_{1} y_{2}\left[\operatorname{comp}(x)\left(y_{1}\right) \wedge y_{2} \sqsubseteq y_{1} \rightarrow \operatorname{comp}(x)\left(y_{2}\right)\right]$

Postulate (3) states that all subparts of a realization of a given kind are also realizations of that kind. ${ }^{3}$ From this postulate it follows, for example, that if $e$ is a process of John running along the river which has a realization $e_{1}$ lasting ten minutes, and if $e_{2}$ is a subevent of $e_{1}$ - the first half, say - then $e_{2}$ is also a realization of $e$. As such, this postulate may be used to make John ran along the river for ten minutes entail John ran along the river for five minutes, in contrast to the pair John ran to the museum in ten minutes and John ran to the museum in five minutes.

In order to resolve the imperfective paradox, we may extend White (1993) by adding a mapping from events to processes (whose realizations need not terminate in the same way), as well as a means for distinguishing actual and hypothetical events. To do the former, we may axiomatize comp's inverse mapping - Jackendoff's ground-from (gr) - again in terms of Krifka's part-of relation. This is shown below:

(4) $\forall x y_{1} y_{2}\left[\operatorname{gr}\left(y_{1}\right)(x) \wedge \operatorname{comp}(x)\left(y_{2}\right) \rightarrow y_{2} \sqsubseteq y_{1}\right]$

Postulate (4) simply requires that all the realizations $e_{2}$ of a process $e$ which is 'ground from' an event $e_{1}$ must be subevents of $e_{1}$ (and likewise, mutatis mutandis, for substances and things). As the realizations $e_{2}$ of $e$ may be proper subevents of $e_{1}$, the relation $\mathbf{g r}$ provides a means for accessing subevents of $e_{1}$ with alternate terminations.

To distinguish those events which actually occur from those that are merely hypothetical, we may simply introduce a special predicate Actual, which we require to preserve the part-of relation only in the downwards direction:

(5) $\forall x y[\operatorname{Actual}(x) \wedge y \sqsubseteq x \rightarrow \operatorname{Actual}(y)]$

Postulate (5) is necessary to get John stopped running to the museum after ten minutes to entail John ran for ten minutes as well as John ran for nine minutes, but not John ran for eleven minutes.

At this point we are ready to examine in some detail how the above machinery may be used in resolving the imperfective paradox. Let us assume

\footnotetext{
${ }^{3}$ For the sake of simplicity I will not address the minimal parts problem here.
}

that sentences such as (6) receive compositional translations as in (7):

(6a) John ran to the bridge.

(6b) John stopped running to the bridge.

(7a) $\exists e_{1}$.

$$
\begin{aligned}
& \operatorname{run}^{\prime}(j)\left(e_{1}\right) \wedge \text { to }^{\prime}\left(\text { the }^{\prime}\left(\operatorname{bridge}^{\prime}\right)\right)\left(\tau_{\mathrm{s}}\left(e_{1}\right)\right) \wedge \\
& \operatorname{Actual}\left(e_{1}\right)
\end{aligned}
$$

(7b) $\exists e e_{1} e_{2} e_{3}$.

$$
\begin{aligned}
& \operatorname{run}^{\prime}(j)\left(e_{1}\right) \wedge \text { to }^{\prime}\left(\text { the }^{\prime}\left(\text { bridge }^{\prime}\right)\right)\left(\tau_{\mathbf{s}}\left(e_{1}\right)\right) \wedge \\
& \operatorname{gr}\left(e_{1}\right)(e) \wedge \operatorname{comp}(e)\left(e_{2}\right) \wedge \operatorname{stop}^{\prime}\left(e_{2}\right)\left(e_{3}\right) \wedge \\
& \text { Actual }\left(e_{3}\right)
\end{aligned}
$$

In (7), $e_{1}$ is an event of John running to the bridge. ${ }^{4}$ In (7a), this event is asserted to be actual; in $(7 \mathrm{~b})$, in contrast, the progressive morphology on run triggers the introduction of $\mathbf{g r}$, which maps $e_{1}$ to the process $e .^{5}$ It is this process which $e_{3}$ is an event of stopping: following Jackendoff (1991), this is represented here by introducing an event $e_{2}$ composed of $e$ which has $e_{3}$ as its stopping point. Naturally enough, we may expect the actuality of $e_{3}$ to entail the actuality of $e_{2}$, and thus all subevents of $e_{2}$. Nevertheless, the actuality of $e_{1}$ does not follow, as Postulate (4) permits $e_{2}$ to be a proper subpart of $e_{1}$ (which is pragmatically the most likely case).

To make the semantics developed so far more concrete, we may now impose a particular interpretation on trajectory-of-motion events, namely one in which these are modeled as continuous functions from times to locations of the object in motion. Depending on how we model objects and locations, we of course arrive at interpretations of varying complexity. In what follows we focus only on the simplest such interpretation, which takes both to be points.

Note that by assuming the preceding interpretation of trajectory-of-motion events, we may interpret the relation $\sqsubseteq$ as the relation continuoussubset. Furthermore, we may also interpret processes as sets of events closed under the $\sqsubseteq$ relation; this then permits comp to be interpreted as element-of, and gr (for events) as mapping an event to the smallest process containing it. Before continuing, we may observe that this interpretation does indeed satisfy Postulates (3) and (4).

\section{Application}

While the above interpretation of trajectory-ofmotion events forces one to abstract away from

\footnotetext{
${ }^{4}$ The spatial trace function $\tau_{s}$ maps eventualities to their trajectories (cf. White 1993).

${ }^{5} \mathrm{Much}$ as in Moens and Steedman (1988) and Jackendoff (1991), the introduction of $\mathbf{g r}$ is necessary to avoid having an ill-sorted formula.
} 
the manner of motion supplied by a verb, it does nevertheless permit one to consider factors such as the normal speed as well as the meanings of the prepositions to, towards, etc. By making two additional restrictive assumptions, namely that these events be of constant velocity and in one dimension, I have been able to construct and implement an algorithm which determines whether a specified sequence of such events is or is not possible under certain situationally supplied constraints. These constraints include the locations of various landmarks (assumed to remain stationary) and the minimum, maximum, and normal rates associated with various manners of motion (e.g. running, jogging) for a given individual.

The algorithm takes an input string and compositionally derives a sequence of logical forms (one for each sentence) using a simple categorial grammar (most of which appears in White 1993). A special-purpose procedure is then used to instantiate the described sequence of events as a constraint optimization problem; note that although this procedure is quite ad-hoc, the constraints are represented in a declarative, hierarchical fashion (cf. White 1993). If the constraint optimization problem has a solution, it is found using a slightly modified version of the constraint satisfaction procedure built into SCREamer, Siskind and McAllester's (1993) portable, efficient version of nondeterministic Common Lisp. ${ }^{6}$

As an example of an impossible description, let us consider the sequence of events described below:

(8) Guy started jogging eastwards along the river. 25 minutes later he reached the cafe / the museum\}.

If we assume that the user specifies the cafe and the museum to be 5 and $10 \mathrm{~km}$, respectively, from the implicit starting point, and that the rates specified for Guy are those of a serious but not superhuman athlete, then the algorithm will only find a solution for the first case $(10 \mathrm{~km}$ in 25 minutes is too much to expect.) Now, by reasoning about subevents - here, subsegments of lines in spacetime - the program exhibits the same behavior with the pair in (9):

(9) Guy started jogging to the bar. 25 minutes later he reached $\{$ the cafe / the museum\}.

Since Guy jogging to the cafe is accepted as a possible proper subevent of Guy jogging to the

\footnotetext{
${ }^{6}$ The constraint optimization problem is split into two constraint satisfaction problems, namely finding the smallest consistent value of a cost variable and then finding consistent values for the rest of the variables.
}

bar (assuming the bar is further east than the other landmarks), example (9) shows how the present approach successfully avoids the imperfective paradox; since Guy jogging to the museum (in 25 minutes) is not accepted as a possible subevent, example (9) likewise shows how the present approach extends and refines those of Moens and Steedman and Jackendoff vis-a-vis the subevent relation. ${ }^{7}$

\section{Future Work}

The algorithm as implemented functions only under a number of quite restrictive assumptions, and suffers from a rather ad-hoc use of the derived logical forms. In future work I intend to extend the algorithm beyond the unidimensional and constant velocity cases considered so far, and to investigate incorporating the present treatment into the Interpretation as Abduction approach advocated by Hobbs et. al. (1993).

\section{References}

[1] Emmon Bach. The algebra of events. Linguistics and Philosophy, 1986.

[2] David R. Dowty. Word Meaning and Montague Grammar. Reidel, 1979.

[3] Kurt Eberle. Eventualities in natural language understanding systems. In Sorts and Types in Artificial Intelligence. Springer Verlag, 1990.

[4] Christopher Habel. Propositional and depictorial representations of spatial knowledge: The case of path concepts. In Natural Language and Logic. Springer Verlag, 1990. Lecture Notes in Artificial Intelligence.

[5] Erhard Hinrichs. A Compositional Semantics for Aktionsarten and NP Reference in English. PhD thesis, The Ohio State University, 1985.

[6] Jerry Hobbs, Mark Stickel, Douglas Appelt, and Paul Martin. Interpretation as abduction, 1993. To appear in Artificial Intelligence Journal.

[7] Ray Jackendoff. Parts and boundaries. Cognition, 41:945, 1991 .

[8] Manfred Krifka. Thematic relations as links between nominal reference and temporal constitution. In Ivan A. Sag and Anna Szabolcsi, editors, Lexical Matters. CSLI, 1992.

[9] Marc Moens and Mark Steedman. Temporal ontology and temporal reference. Computational Linguistics, June 1988.

[10] Jeffrey Mark Siskind and David Allen McAllester. Nondeterministic lisp as a substrate for constraint logic programming. To appear in AAAl-93, 1993.

[11] H. J. Verkuyl. Aspectual classes and aspectual composition. Linguistics and Philosophy, 12(1), 1989.

[12] Michael White. Delimitedness and trajectory-of-motion events. In Proceedings of the Sixth Conference of the European Chapter of the Association for Computational Linguistics (EACL '93), 1993.

\footnotetext{
${ }^{7}$ It is worth noting that the constant velocity restrictive assumption makes start running to and start running towards synonymous, which is not the case in general (cf. Habel 1990).
} 